\title{
Chikungunya virus adaptation to Aedes albopictus mosquitoes does not correlate with acquisition of cholesterol dependence or decreased $\mathrm{pH}$ threshold for fusion reaction
}

\author{
Konstantin A Tsetsarkin ${ }^{1 *}$, Charles E McGee ${ }^{2}$ and Stephen Higgs ${ }^{1}$
}

\begin{abstract}
Background: Chikungunya virus (CHIKV) is a mosquito transmitted alphavirus that recently caused several large scale outbreaks/epidemics of arthritic disease in tropics of Africa, Indian Ocean basin and South-East Asia. This reemergence event was facilitated by genetic adaptation (E1-A226V substitution) of CHIKV to a newly significant mosquito vector for this virus; Aedes albopictus. However, the molecular mechanism explaining the positive effect of the E1-A226V mutation on CHIKV fitness in this vector remains largely unknown. Previously we demonstrated that the E1-A226V substitution is also associated with attenuated CHIKV growth in cells depleted by cholesterol.

Methods: In this study, using a panel of CHIKV clones that varies in sensitivity to cholesterol, we investigated the possible relationship between cholesterol dependence and Ae. albopictus infectivity.

Results: We demonstrated that there is no clear mechanistic correlation between these two phenotypes. We also showed that the E1-A226V mutation increases the $\mathrm{pH}$ dependence of the CHIKV fusion reaction; however, subsequent genetic analysis failed to support an association between CHIKV dependency on lower $\mathrm{pH}$, and mosquito infectivity phenotypes.
\end{abstract}

Conclusion: the E1-A226V mutation probably acts at different steps of the CHIKV life cycle, affecting multiple functions of the virus.

Keywords: Chikungunya virus, Aedes albopictus, cholesterol, pH threshold of fusion

\section{Introduction}

Chikungunya virus (CHIKV), an arthritogenic alphavirus, has received global attention due to the series of recent large-scale outbreaks in different parts of the world including Africa, Indian Ocean Islands, India and countries of South-East Asia [1,2]. The virus was also introduced with viremic travelers into Europe where it caused, for the first time, autonomously transmitted outbreaks in 2007 [3] and 2010 [4]. The global expansion of CHIKV was at least partially attributed to the viral adaptation to a new mosquito vector Aedes (Ae.) albopictus, which facilitated CHIKV transmission in the regions

\footnotetext{
* Correspondence: kotsetsa@utmb.edu

'Department of Pathology, University of Texas Medical Branch, Galveston, Texas, USA

Full list of author information is available at the end of the article
}

that lack typical vector Ae. aegypti [5-10]. Phylogenetic studies have identified a specific mutation (E1-A226V) in the E1 glycoprotein of CHIKV strains circulating in the areas where Ae. albopictus serves as primary virus vector $[6,8,9,11-13]$. Additionally, laboratory studies confirmed that the E1-A226V substitution leads to increased CHIKV infectivity and is associated with more efficient dissemination and transmission by Ae. albopictus $[14,15]$. We also demonstrated that midgut epithelial cells of Ae. albopictus appear to be the primary target cells where this mutation has the most profound effect [16]. However, the molecular mechanism underlying the positive effect of E1-A226V on CHIKV infectivity for midgut cells of Ae. albopictus remains largely unknown.

The envelope of CHIKV consists of 240 copies of membrane-embedded E2-E1 heterodimers, which

\section{Ciomed Central}


surround the nucleocapsid core. Cellular entry is mediated by the interaction of E2 glycoprotein with an unidentified receptor, followed by endocytosis of the virion-receptor complex into endosomal compartments. Acidification of endosomes subsequently triggers a cascade of events that culminates in E1 activation and viral fusion reaction [17-19]. Since the mutation responsible for adaptation of CHIKV to Ae. albopictus occurs in the E1 protein; a receptor-mediated explanation of its effect on viral fitness in this mosquito seems implausible, suggesting that this mutation acts at steps in the virus life cycle following receptor-mediated endocytosis.

Previously it has been shown that mutations at the same position of the E1 protein (E1-226) of other alphaviruses; Semliki Forest virus (SFV) and Sindbis virus (SINV), can modulate the cholesterol requirements for viral entry into, and exit from, cells [20-23]. Thus, the single amino acid substitution E1-P226S in SFV and triple substitution at (E1-A226S, E1-K227G, E1-N228M) in SINV was sufficient to significantly increase virus production in, virus infectivity to, and virus exit from cholesterol-depleted Ae albopictus (C6/36) cells [22,23]. Position E1-226 is located at the tip of the ij loop of the E1 protein of alphaviruses [24,25] and it was hypothesized that the presence or absence of cholesterol in target membranes is detected by residues in this loop, which influences the kinetics of E1 conformational changes during fusion [26]. Interestingly, in a study using Venezuelan equine encephalitis virus (VEEV)enveloped pseudo-typed murine leukemia virus, it was shown that the entry of VEEV into cells is resistant to cholesterol depletion of the mammalian cells [27], suggesting that cholesterol dependence is not absolute and probably depends on particular virus/host species relationships.

Previously, we demonstrated that the E1-A226V mutation that confers increased fitness of CHIKV in Ae. albopictus is also associated with CHIKV dependence for cholesterol for growth in mosquito derived cell lines. Thus, the viruses that have alanine at E1-226 grew to 4$5 \log _{10} \mathrm{TCID}_{50} / \mathrm{mL}$ higher titers in cholesterol-depleted C6/36 cells as compared to viruses that have valine at this position [15]. However, no difference in growth kinetics between these viruses was detected in standard C6/36 cells, suggesting that dependence for cholesterol might be directly connected to the mosquito infectivity phenotype.

In this study, to investigate possible relationship between cholesterol dependence of CHIKV and Ae. albopictus infectivity, we generated a panel of CHIKV clones that varies in sensitivity to cholesterol and evaluated their relative infectivites for Ae. albopictus mosquitoes.

\section{Materials and methods}

\section{Plasmids and cells}

The plasmids encoding full-length infectious clones of the LR2006 OPY1 strain pCHIKV-LR i.c. (GenBank accession number EU224268), GFP-expressing full length clone pLR-GFP-226V (pCHIKV-LR 5'GFP, GenBank accession number EU224269), pLR-ApaI-226V and pLR-226A have been previously described $[15,28]$. A series of point mutations at E1-226 (S, T, G, I, P, F, $\mathrm{M}, \mathrm{H}, \mathrm{L}$ ) was introduced into pCHIKV-LR i.c. using standard molecular biology methods that resulted in clones designated as pLR-226X, where X corresponds to one of residues to be introduced. To introduce $\mathrm{S}, \mathrm{T}, \mathrm{G}$, I, P, F, M, H, and L residues individually at E1-226 in the background of eGFP-expressing CHIKV pLR-GFP226V [15], the DNA fragment of 4709 nt. was generated by digestion of pLR-GFP-226V with AgeI and XhoI. This fragment was cloned into each of pLR-226X plasmids by $A g e \mathrm{I}$ and $\mathrm{XhoI}$ sites. The resultant plasmids were designated pLR-GFP-226X. Presence of the eGFP gene in the plasmid was confirmed by restriction digestion analysis.

A plasmid encoding the pCHIKV-LR i.c. containing mutations identified in the cholesterol-independent plaque purified clone of LR-ApaI-226V (Clone\#1) [see below; adaptation of LR-ApaI-226V to cholesteroldepleted C6/36 cells] was generated by cloning of two cDNA fragments into pCHIKV-LR 3'GFP [28]. Fragment 1 (1386 nt) was amplified based on RNA isolated form Clone\# 1 virus using CHIKV specific primers, and digested with $\mathrm{XhoI}$ and $\mathrm{Kp} n \mathrm{I}$ restrictases. Fragment 2 (961 nt) was generated by digestion of pLR-GFP-226V with KpnI and PacI. Fragments 1 and 2 were simultaneously ligated and cloned by $\mathrm{XhoI}$ and PacI sites into pCHIKV-LR 3'GFP. The resultant plasmid was named pLR-Cl\#1. To introduce a single point mutation, C10660A, into pLR-Cl\#1, two DNA fragments of 1082 nt. and 1249 nt. were generated by digestion of pLR$\mathrm{Cl} \# 1$ with $\mathrm{XhoI}$ and EcoRV and digestion of pLR-GFP226V with EcoRV and PacI restrictases, respectively. Both DNA fragments were cloned simultaneously into pCHIKV-LR 3'GFP by XhoI and PacI sites. The eGFP expressing version of this plasmid was generated using the same strategy as was described for pLR-GFP-226X constructs. The resultant clone was designated as pLRGFP-Cl\#1-10660A and sequenced.

The presence of correct nucleotide substitutions leading to the desired amino acid mutations at specific position of E1 gene was confirmed by sequencing analysis. All clones possessed mutations of interest and no additional substitutions were detected. Plasmids were propagated in MC1061 strain of E.coli and purified using the QIAprep Spin Miniprep Kit (Qiagen, Valencia, CA) 
following manufacturer's protocol. Detailed information for all constructs is available from authors upon request.

BHK-21 (Baby Hamsters Kidney) and Vero (African green monkey kidney) cells were maintained at $37^{\circ} \mathrm{C}$ with $5 \% \mathrm{CO}_{2}$. BHK-21 were propagated in MEM alpha (Invitrogen, Carlsbad, CA) supplemented with 10\% FBS and $1 \times$ MEM vitamin solution (Invitrogen, Carlsbad, CA). Vero cells were maintained in MEM (Invitrogen, Carlsbad, CA) supplemented with 5\% BGS and $1 \times$ MEM non-essential amino acids solution (Invitrogen, Carlsbad, CA). C6/36 cells (Ae. albopictus) were maintained at $28^{\circ} \mathrm{C}$ without $\mathrm{CO}_{2}$ in Leibovitz L-15 (L-15) medium supplemented with $10 \%$ FBS.

\section{Rescue of infectious viruses from infectious clones}

One microgram of plasmid DNA was linearized with NotI restrictase and was used as a template for in-vitro transcription reaction using the mMESSAGE mMACHINE kit (Ambion, Austin, TX) according to the manufacturer's protocol. Ten micrograms of in-vitro transcribed RNA were electroporated without further purification into $10^{7}$ BHK-21 cells using Gene Pulser Xcell electroporation system (Biorad, Hercules, CA) and preset BHK-21 conditions as described previously [29]. Electroporated cells were maintained in $75 \mathrm{~cm}^{2}$ tissue culture flasks at $37^{\circ} \mathrm{C}$ in $15 \mathrm{~mL}$ of L-15 medium. Cell culture supernatants were collected at 24 and $48 \mathrm{~h}$ postinfection (hpi), and stored at $-80^{\circ} \mathrm{C}$. Viral titers were determined by titration of the infectious samples on Vero cells in 96-well plate using standard techniques [29]. Titers were expressed as tissue culture infectious dose $50 \%$ endpoint per milliliter of media $\left(\log _{10} \mathrm{~T}\right.$ $\mathrm{CID}_{50} / \mathrm{mL}$ ) [29].

Specific infectivity of electroporated RNA was determined as previously described [28]. Briefly, $1 \times 10^{5}$ of electroplated BHK-21 cells were ten-fold diluted and transferred onto sub-confluent monolayers $\left(1 \times 10^{6}\right.$ cells/well) of uninfected Vero cells in six-well plates [28]. Cells were incubated for $2 \mathrm{~h}$ to allow attachment to the plate, and the media was replaced with $2 \mathrm{~mL}$ of $0.5 \%$ agarose in MEM supplemented with $3.3 \%$ FBS. Plates were incubated for 48-96 h until visible plaques developed. The results (specific infectivity values) were expressed as pfu/1 $\mu \mathrm{g}$ of electroporated RNA [Additional file 1, Table S1 and Table S2].

\section{In vitro growth of CHIKV in standard and cholesterol- depleted $\mathrm{C} 6 / 36$ cells}

To investigate if the mutations in the E1 protein influenced cholesterol dependence of CHIKV, cholesteroldepleted C6/36 cells were generated by five passages in MEM alpha medium containing 10\% FBS treated with $2 \%$ CAB-O-Sil (Acros Organics) for $12 \mathrm{~h}$ at room temperature as previously described [30]. CAB-O-Sil is a hydrated colloidal silica that adsorbs lipoproteins/cholesterol and allows removal of cholesterol from biological solutions. CHIKV growth curves were determined by infecting cholesterol-depleted and normal C6/36 cells duplicates in $25 \mathrm{~cm}^{2}$ flasks at multiplicities of infection (MOI) of 0.1 and 1.0, respectively, by rocking for $1 \mathrm{~h}$ at $25^{\circ} \mathrm{C}$. The cells were washed three times with medium and $5 \mathrm{~mL}$ of fresh L-15 or MEM alpha medium supplied with $10 \%$ of standard or CAB-O-Sil treated FBS was added to the flask. Five hundred microliters of medium was collected from each flask at the indicated times post-infection, and medium volume was adjusted by adding $0.5 \mathrm{~mL}$ of appropriate medium. Viral titers were determined by titration of cell culture samples on Vero cells.

\section{C6/36 cells infectivity assay}

Primary infections of cholesterol-depleted C6/36 cells with CHIKV containing specific mutations in the E1 glycoprotein were quantified using an infectious centers assay. Confluent monolayers of cholesterol-depleted C6/ 36 cells in 96 well plates were infected with $10 \mu \mathrm{L}$ of serial ten-fold dilutions of eGFP expressing CHIK viruses, for $1 \mathrm{~h}$ at room temperature. The virus dilutions were made in MEM alpha medium containing 10\% FBS treated with $2 \%$ CAB-O-Sil. Following infection, $100 \mu \mathrm{L}$ of MEM alpha medium containing $10 \%$ cholesteroldepleted FBS and $0.8 \%$ of carboxy methyl cellulose (which prevents spread of viruses beyond the initial focus of infection) were added to each well. Plates were incubated for $20 \mathrm{~h}$ at $30^{\circ} \mathrm{C}$. Experiments were performed in triplicates, and eGFP expressing foci were counted and results were normalized to $10^{6}$ infectious centers/ $\mathrm{mL}$ for standard $\mathrm{C6} / 36$ cells.

\section{Adaptation of LR-Apal-226V to cholesterol-depleted C6/ 36 cells}

LR-ApaI-226V virus was passed four times in cholesterol-depleted C6/36 cells maintained in L-15 medium. At first passage, a confluent monolayer of cholesteroldepleted C6/36 in $25 \mathrm{~cm}^{2}$ tissue culture flask was infected with LR-ApaI-226V at an MOI of 0.1. At 3 days post infection (dpi), $1 \mathrm{~mL}$ of tissue culture supernatant was used to infect new cholesterol-depleted C6/ 36 in $25 \mathrm{~cm}^{2}$ tissue culture flasks. After the fourth passage in cholesterol-depleted C6/36 cells, tissue culture supernatant was harvested at $2 \mathrm{dpi}$ and stored at $-80^{\circ} \mathrm{C}$ until needed. To obtain the plaque-purified clones, supernatant of the passage four was titrated by plaque assay on Vero cells as previously described [31]. At 2 $\mathrm{dpi}$, ten individual plaques below the agarose were pierced with a $200 \mu \mathrm{L}$ tip and used for infection of standard C6/36 cells in L-15 medium in one well of a 12 well plate. At 2 dpi culture supernatants were harvested, 
titrated and stored at $-80^{\circ} \mathrm{C}$ until needed. The 9500 $11000 \mathrm{nt}$. genome region of the plaque-purified clones was sequenced.

\section{Virus competition assay in cells treated with $\mathrm{NH}_{4} \mathrm{Cl}$}

BHK-21 or C6/36 cells in 12 well plates were maintained in L-15 medium supplied with $10 \%$ FBS. Thirty minutes prior to infection, cell culture media was replaced with $0.5 \mathrm{~mL}$ of the $\mathrm{L}-15$ media containing various amounts of $\mathrm{NH}_{4} \mathrm{Cl}$ (endosomal acidification inhibitor) as indicated (see results). After a 30 min incubation at room temperature, $50 \mu \mathrm{L}$ of a 1:1 mixture of LRApaI-226V and LR-226A virus $\left(10^{7} \mathrm{pfu} / \mathrm{mL}\right)$ was added to each well and infection was allowed to continue for 1 $\mathrm{h}$ at room temperature. Cells were washed once with 0.8 $\mathrm{mL}$ of $\mathrm{L}-15$ media containing $\mathrm{NH}_{4} \mathrm{Cl}$, then $0.8 \mathrm{~mL}$ of fresh L-15 medium containing various amounts of $\mathrm{NH}_{4} \mathrm{Cl}$ was added to each well and cells were incubated for $48 \mathrm{~h}$ at $37^{\circ} \mathrm{C}$ for $\mathrm{BHK}-21$ or $28^{\circ} \mathrm{C}$ for $\mathrm{C} 6 / 36$ cells. Cell culture supernatants were harvested and used for RNA extraction, or titration on Vero cells. Virus RNA was processed as discussed previously [15].

\section{Cell-cell fusion assay}

For comparative studies of the $\mathrm{pH}$ threshold of the $\mathrm{CHIKV}$ fusion reaction, confluent monolayers of C6/36 cells in 24-well plate were infected (MOI of 0.5-1.0) with eGFP expressing CHIKV containing specific mutations in the E1 glycoprotein. Cells were incubated for 24 $\mathrm{h}$ in $0.5 \mathrm{~mL}$ of L-15 medium supplemented with $10 \%$ FBS to allow cell surface expression of viral glycoproteins. Cells were washed once with $0.5 \mathrm{~mL}$ of L-15 and the cell-to-cell fusion reaction was triggered by incubating the cell monolayers for $2 \mathrm{~min}$ at $30^{\circ} \mathrm{C}$ in $1 \mathrm{~mL}$ of pre-warmed $\left(30^{\circ} \mathrm{C}\right) \mathrm{L}-15$ medium whose $\mathrm{pH}$ was previously adjusted to desired values. Fusion reactions were stopped by replacement of the fusion medium with 0.5 $\mathrm{mL}$ of standard L-15 medium ( $\mathrm{pH} 7.4$ ). Cells were incubated for $3 \mathrm{~h}$ at $28^{\circ} \mathrm{C}$ to allow polykaryons to develop. Cells were fixed with $3.5 \%$ formaldehyde and stained with $1 \mu \mathrm{g} / \mathrm{mL}$ of 4',6-diamidino-2-phenylindole (DAPI) to visualize nuclei. Cells were analyzed by fluorescent microscopy and the fusion index (percent of fusion) was calculated as $(1-\mathrm{c} / \mathrm{n}) \times 100 \%$, where $\mathrm{c}$ is the number of eGFP expressing cells, $n$ is the number of nuclei $(n \geq 70)$. Experiments were performed in duplicates for each virus.

\section{Oral infection of mosquitoes}

Ae. albopictus (Galveston strain) were infected in an Arthropod Containment Level 3 (ACL-3) insectary as described previously [32,33]. To estimate the Oral infectious dose $50 \%\left(\mathrm{OID}_{50}\right)$ values, serial ten-fold dilutions of BHK-21 derived viruses were made in L-15 medium followed by mixing equal volume of virus samples with defibrinated sheep blood (Colorado Serum Company, Denver, CO). Blood meals were presented to 4-5 day old female Ae. albopictus (100 mosquitoes per virus dilution) using Hemotek feeding apparatus (Discovery Workshops, Accrington, Lancashire, United Kingdom) as described previously [15]. Fully engorged mosquitoes were sorted on ice and placed in carton boxes for maintenance at $16 / 8 \mathrm{~h} \mathrm{light/dark}$ photoperiod at $28^{\circ} \mathrm{C}$ and $80 \%$ humidity, with a $10 \%$ sucrose solution provided ad libitum. Midguts were dissected at $7 \mathrm{dpi}$ and virusinduced eGFP expression in midgut epithelium was analyzed by fluorescence microscopy. CHIKV infection of mosquitoes was detected by observation of CHIKVexpressed eGFP in epithelial cells. The oral infectious dose $50 \%\left(\mathrm{OID}_{50}\right)$ values and fiducial limits were calculated using PriProbit (version 1.63) and SAS equivalent method assuming normal function distribution and an "all or nothing" response parameter. For stability assays, 3-4 mosquitoes infected with the highest dilution of the selected viruses were triturated in $1 \mathrm{~mL}$ of TRIzol reagent (Invitrogen, Carlsbad, CA) at $7 \mathrm{dpi}$, and total mosquito and viral RNA was extracted according to the manufacturer's instructions. Viral cDNA was amplified, and the region of interest was directly sequenced using an ABI PRISM model 3100 Genetic Analyzer (Applied Biosystems, Foster City, CA).

\section{Results}

To better understand the amino acid requirements at position E1-226 for increased CHIKV midgut infectivity of Ae. albopictus, and to understand how it might correlate with cholesterol dependence of CHIKV, a number of CHIKV containing a single amino acid substitutions at E1-226 were constructed and tested in cholesteroldepleted C6/36 cells and in Ae. albopictus. Initially, mutations encoding S, T, G, and I residues at E1-226 were introduced into the backbone of non-eGFP expressing pCHIKV-LR i.c. Specific infectivity values were comparable for all of the constructs, indicating that introduction of these residues at E1-226 is not lethal for CHIKV replication in BHK-21 cells (Additional file 1, Table S1). Growth kinetics of LR-226S, LR-226T, LR226G and LR-226I were determined in standard and cholesterol-depleted C6/36 cells, and compared with growth of LR-ApaI-226V and LR-226A viruses, which have been tested previously [15]. All viruses grew very efficiently in standard C6/36 cells (Figure 1A), however, in cholesterol-depleted cells only LR-226S demonstrated growth kinetics comparable with LR-226A. The growth of all other viruses was significantly attenuated, the peak titers being 3-4 $\log _{10} \mathrm{TCID}_{50} / \mathrm{mL}$ lower at 24,48 , and $72 \mathrm{hpi}$ as compared with LR-226A and LR-226S (Figure 1B). To further investigate the effects of $S, T, G$ and I 

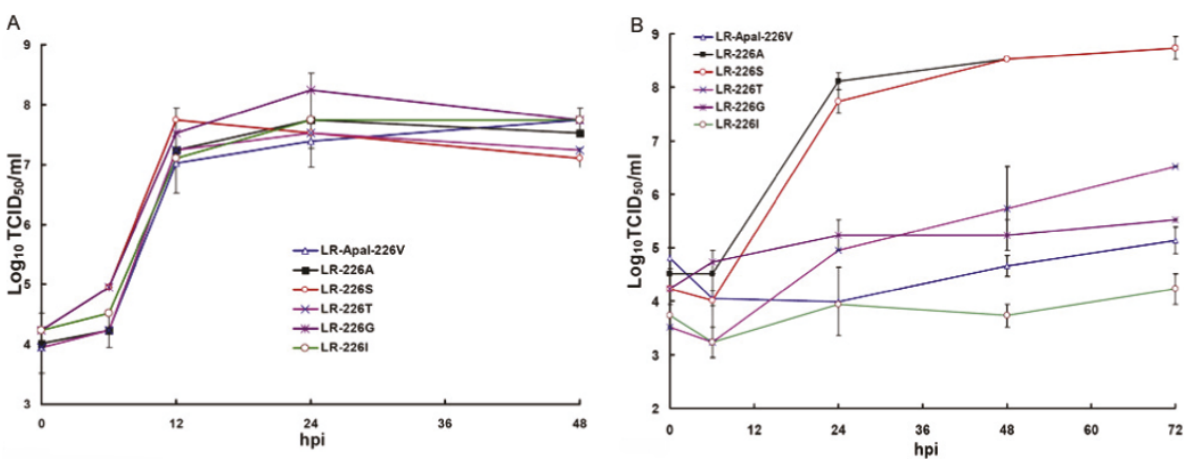

Figure 1 Effect of S, T, G and I residues at E1-226 on growth of CHIKV in standard (A) and cholesterol-depleted (B) C6/36 cells Standard cells were infected at an MOI of 1.0. Cholesterol-depleted cells were infected at an MOI of 0.1. Viral titers are expressed as $\log _{10} T C I D_{50} / \mathrm{mL} \pm$ standard deviation of 2 independent experiments. hpi - hours post-infection.

residues at E1-226 on the CHIKV requirement for cholesterol, the mutations encoding these amino acids were introduced into the backbone of eGFP-expressing i.c. of CHIKV (Additional file 1, Table S2), and then specific infectivity of these viruses were determined in standard and cholesterol-depleted C6/36 cells (Figure 2). Relative infectivity of LR-GFP-226A and LR-GFP-226S for cholesterol-depleted $\mathrm{C} 6 / 36$ was $2-2.5 \log _{10} \mathrm{IC} / \mathrm{mL}$ higher than the infectivity of CHIKV with V, G, or I residues. This is in agreement with the growth kinetics of these viruses in cholesterol-depleted C6/36 cells, indicating that cell infectivity of E1-226V, E1-226G and E1-226I containing virus is much more dependent on the presence of cholesterol than infectivity of E1-226A and E1$226 \mathrm{~S}$ viruses. Interestingly, the cell infectivity of LRGFP-226T virus was significantly lower ( $<00.01 \mathrm{Stu}-$ dent's t-test) than LR-GFP-226A and LR-GFP-226S viruses, but was significantly higher than that of LRGFP-226V, LR-GFP-226G and LR-GFP-226I viruses, indicating CHIKV dependence for cholesterol can be

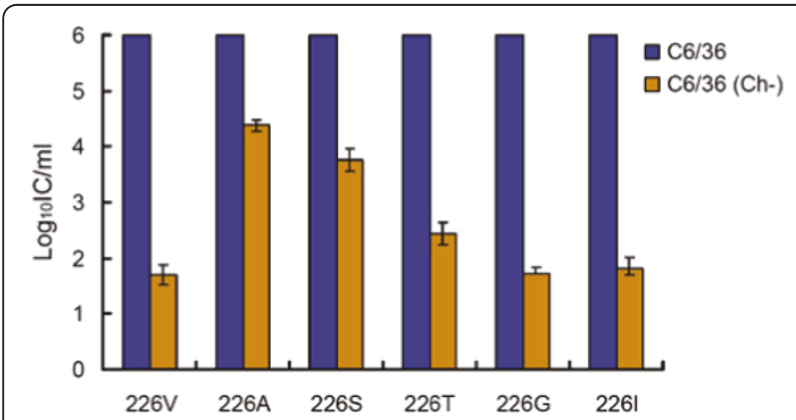

Figure 2 Effect of S, T, G and I residues at E1-226 on CHIKV infectivity for cholesterol-depleted $\mathrm{C} 6 / 36$ cells. Standard (blue bars) and cholesterol-depleted (yellow bars) C6/36 cells were infected with serial dilutions of eGFP expressing CHIKV containing indicated residues at E1-226. Results are normalized for $10^{6}$ viral infections of standard C6/36 cells. Data indicate an average of three experiments \pm standard deviation. IC-infectious center. expressed as a spectrum of different phenotypes (Figure 2).

To investigate the relationship between CHIKV dependence for cholesterol and CHIKV infectivity for midgut cells of Ae. albopictus mosquitoes, serial dilutions of LR-GFP-226S, LR-GFP-226T, LR-GFP-226G and LR-GFP-226I viruses were orally presented to $A e$. albopictus mosquitoes. CHIKV infectivity was analyzed by the expression of eGFP in the midgut cells at $7 \mathrm{dpi}$ (Table 1). OID 50 values of LR-GFP-226S, LR-GFP-226T and LR-GFP-226G in Ae. albopictus were between 5.35 and 5.94 $\log _{10} \mathrm{TCID}_{50} / \mathrm{mL}$, which were not significantly different from the OID $_{50}$ value of LR-GFP-226A virus (5.45 $\log _{10} \mathrm{TCID}_{50} / \mathrm{mL}$ ). This indicates that cholesterol dependence of CHIKV does not directly correlate with an increase in midgut infectivity. LR-GFP-226T and LRGFP-226G both were significantly more cholesteroldependent as compared to LR-GFP-226A and LR-GFP226S; however, all four viruses demonstrated almost identical infectivity for Ae. albopictus mosquitoes. Infectivity of LR-GFP-226I was almost identical to infectivity of epidemic CHIKV containing the E1-226V residue ( $\mathrm{p}$ $<0.1$ ). Both valine (V) and isoleucine (I) have additional methyl and ethylene groups in their side chains as compared to alanine or glycine, which are probably required for an increase in CHIKV infectivity for midgut cells of Ae. albopictus. The presence of a hydroxyl group in the side chains of serine and threonine apparently has a negative effect on CHIKV infectivity to Ae. albopictus.

To further investigate the amino acid requirement at position E1-226 for increased CHIKV midgut infectivity and cholesterol dependence, mutations encoding P, F, $\mathrm{M}, \mathrm{H}$ and $\mathrm{L}$ at E1-226 were introduced into pCHIKVLR i.c. All constructs had comparable specific infectivity values of in vitro transcribed RNAs and produced moderate to high virus titers in BHK-21 cells (Additional file 1 , Table 1), indicating that these residues are not lethal for CHIKV, at least in BHK-21 cells. However, 
Table 1 Effect of mutations at E1-226 and (E1-66/E1-70) on CHIKV infectivity for Ae. albopictus midgut cells

\begin{tabular}{lcccccl}
\hline Backbone & E1 226 & Exp & $\mathbf{N ~} \mathbf{~ m}$ & OID $_{50}$ & FL $_{95}$ & p-value \\
\hline LR-GFP-226A & $\mathrm{A}$ & Com & 194 & 5.45 & $\mathrm{NG}$ & - \\
LR-GFP-226V & $\mathrm{V}$ & Com & 261 & 3.52 & $\mathrm{NG}$ & $<0.01$ \\
LR-GFP-226S & $\mathrm{S}$ & 1 & 70 & 5.94 & $5.68-6.24$ & $>0.1$ \\
& & 2 & 131 & 5.68 & $5.45-5.92$ & $>0.1$ \\
LR-GFP-226T & $\mathrm{T}$ & 1 & 72 & 5.35 & $5.09-5.61$ & $>0.1$ \\
LR-GFP-226G & $\mathrm{G}$ & 1 & 107 & 5.93 & $5.67-6.19$ & $>0.1$ \\
LR-GFP-226I & $\mathrm{I}$ & 1 & 98 & 3.54 & $1.94-4.02$ & $<0.01$ \\
LR-GFP-226P & $\mathrm{P}$ & 1 & 98 & 4.78 & $4.47-5.10$ & $<0.05$ \\
& & 2 & 137 & 4.86 & $4.43-5.11$ & $<0.05$ \\
LR-GFP-226F & $\mathrm{F}$ & 1 & 112 & 5.62 & $5.32-5.99$ & $>0.1$ \\
LR-GFP-226M & $\mathrm{M}$ & 1 & 117 & 3.30 & $2.49-3.68$ & $<0.01$ \\
LR-GFP-226H & $\mathrm{H}$ & 1 & 119 & 4.69 & $4.44-4.91$ & $<0.05$ \\
& & 2 & 99 & 4.68 & $4.40-4.93$ & $<0.05$ \\
LR-GFP-226L & $\mathrm{L}$ & 1 & 105 & 3.85 & $3.57-4.16$ & $<0.01$ \\
LR-GFP-Cl\#1-10660A & $\mathrm{V}$ & 1 & 80 & 3.41 & $3.32-3.50$ & $<0.01$ \\
\hline
\end{tabular}

E1-226 - residues at position E1-226. Exp - experiment number. $\mathrm{N} \mathrm{m}$ - number of mosquitoes used to estimate $\mathrm{OID}_{50}$ value. $\mathrm{OID}_{50}$ - oral infectious dose $50 \%$, expressed as $\log _{10} \mathrm{TCID}_{50} / \mathrm{mL}$. $\mathrm{FL}_{95}-95 \%$ fiducial limits for $\mathrm{OID}_{50}$ values expressed as $\log _{10} \mathrm{TCID}_{50} / \mathrm{mL}$. p-value - comparison of statistical significance of difference in $\mathrm{OID}_{50}$ values for CHIKV with indicated residues at E1-226 and $\mathrm{OID}_{50}$ value of LR-GFP-226A. Comb - combined summary of two independent experiments. NG - data not given.

constructs with $\mathrm{F}, \mathrm{M}$ and $\mathrm{H}$ residues developed plaques only at 72 hpe in Vero cells, and LR-226L developed plaques at $96 \mathrm{hpe}$, indicating an attenuated phenotype.

Growth kinetics of all these viruses (except LR-226L) were comparable in standard C6/36 cells (Figure 3A). However, growth of these viruses was significantly inhibited at 24, 48 and $72 \mathrm{hpi}$ in cholesterol-depleted cells as compared to LR-226A or LR226S viruses (Figure 3B) (p $<0.05)$. Specific infectivity of eGFP-expressing viruses encoding $\mathrm{P}, \mathrm{F}, \mathrm{M}$, or $\mathrm{H}$ at the position E1-226 in cholesterol-depleted C6/36 cells were significantly lower as compared to the infectivity of LR-GFP-226A; however, infectivity of LR-GFP-226P and LR-GFP-226F were significantly higher than that of LR-GFP-226V virus, which agrees with the growth kinetics results (Figure 4). The $\mathrm{OID}_{50}$ of LR-GFP-226F for Ae. albopictus was similar to the OID $_{50}$ value of LR-GFP-226A ( $p>0.1$ ), indicating that E1-226F does not lead to increased CHIKV infectivity (Table 1). Since LR-GFP-226F was found to be much more cholesterol-dependent as compared to LR-GFP$226 \mathrm{~A}$, this experiment further supported the earlier observation that dependence for cholesterol does not correlate with increased Ae. albopictus midgut infectivity. The OID $_{50}$ values of LR-GFP-226M and LR-GFP$226 \mathrm{~L}$ were indistinguishable as compared with the LRGFP-226V and LR-GFP-226I viruses (Table 1), suggesting that V, I, M and L all share similar structural properties important for CHIKV infectivity for Ae. albopictus. Interestingly, LR-GFP-226P and LR-GFP$226 \mathrm{H}$ demonstrated an intermediate infectivity phenotype, which was statistically different from highly infectious viruses such as LR-GFP-226 [V or I] $(\mathrm{p}<0.05)$ and/or low infectivity viruses such as LR-GFP-226 [A or $\mathrm{S}](\mathrm{p}<0.05)$.

The stability of LR-GFP-226 [I, F, M, H, L] was also analyzed (Additional file 1, Table S3). All mosquitoes infected with CHIKV containing either I, F, M or $\mathrm{H}$ residues were found to replicate RNA encoding the I, F, $\mathrm{M}$, and $\mathrm{H}$ amino acids at E1-226, respectively, indicating that these viruses remain stable after mosquito infection. Two of four mosquitoes infected with virus derived from the pLR-GFP-226L construct contained RNA encoding only the E1-226L residue. However, sequence results from the remaining two mosquitoes indicated that two RNA species (encoding E1-226L and E1-226P) were simultaneously present in these mosquitoes. These data indicate that LR-GFP-226L is unstable and evolves quickly to eliminate the attenuating E1-226L residue (Additional file 1, Table S3).
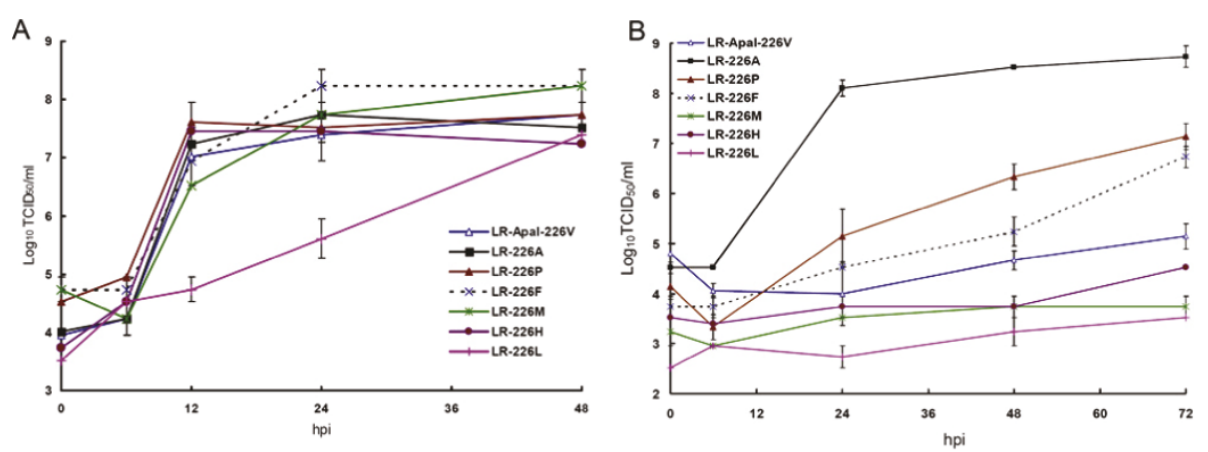

Figure 3 Effect of P, F, M, H and L residues at E1-226 on growth of CHIKV in standard (A) and cholesterol-depleted (B) C6/36 cells Standard cells were infected at an $\mathrm{MOI}$ of 1.0. Cholesterol-depleted cells were infected at an $\mathrm{MOI}$ of 0.1. Viral titers are expressed as $\log _{10} \mathrm{TCID}{ }_{50} / \mathrm{mL} \pm$ standard deviation of 2 independent experiments. 


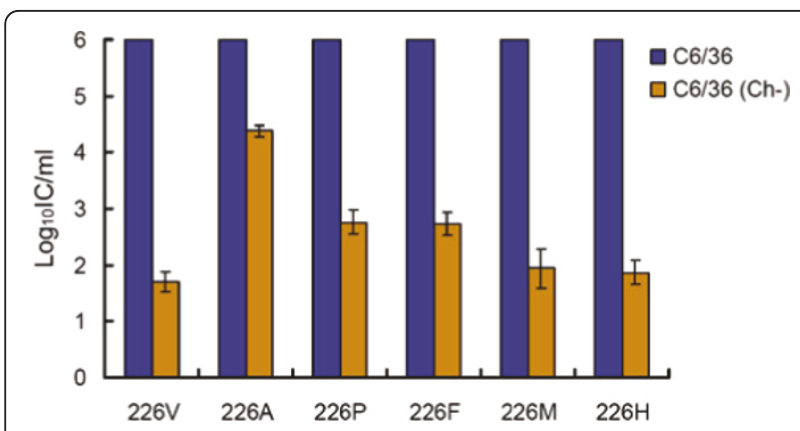

Figure 4 Effect of $P, F, M$ and $H$ residues at E1-226 on CHIKV infectivity for cholesterol-depleted $\mathrm{C} 6 / 36$ cells. Standard (blue bars) and cholesterol-depleted (yellow bars) C6/36 cells were infected with serial dilutions of eGFP expressing CHIKV containing indicated residues at E1-226. Results are normalized for $10^{6}$ viral infections of standard C6/36 cells. Data indicate an average of three experiments \pm standard deviation

\section{Mutations in other CHIKV genome regions that control cholesterol dependence of CHIKV}

The mutagenesis analysis of the position E1-226 in CHIKV showed that all of the residues that confer an increased infectivity phenotype in Ae. albopictus (V, I, $\mathrm{M}$ and $\mathrm{L}$ ) also associated with increased dependence for cholesterol in the target membrane. To investigate if increased CHIKV dependence for cholesterol is absolutely required for increased infectivity in Ae. albopictus, the LR-ApaI-226V virus, with the E1-226V residue conferring high cholesterol dependence was used to select mutations that can control cholesterol dependence of CHIKV. LR-ApaI-226V was passed four times in cholesterol-depleted C6/36 cells. After the first passage, the virus titer was $5.52 \log _{10} \mathrm{TCID}_{50} / \mathrm{mL}$ at $3 \mathrm{dpi}$, but at 2 dpi of passage four, the titer was $7.95 \log _{10} \mathrm{TCID}_{50} / \mathrm{mL}$, indicating successful adaptation to cholesterol-depleted cells. The passage four virus (P4) was plaque purified on Vero cells and 10 individual clones were selected at 2 dpi. Clones were propagated once in C6/36 cells, and the 9500-11000 nt. genome region was sequenced (Additional file 1, Table S4). Seven of 10 sequenced cloned viruses contained the E1-V226A mutation. The second most common variant (3 of 10) contained three nucleotide substitutions $(10189 \mathrm{G} \rightarrow \mathrm{T} ; 10201 \mathrm{G} \rightarrow \mathrm{A}$; $10660 \mathrm{~A} \rightarrow \mathrm{C}$ ); encoding two novel mutations (E1-A66S and E1-D70N). In two clones (\#1 and \#5) these three changes were the only found mutations, but in clone \#7, these mutations were accompanied by an E1-V226A substitution. The least common variant (1 out of 10) contained two nucleotide substitutions $(10659 \mathrm{G} \rightarrow \mathrm{T}$ and $10970 \mathrm{G} \rightarrow \mathrm{A}$ ) leading to two amino acid changes (E1-Q222H and E1-G326D) was excluded form further analysis.

The mutations $[10189 \mathrm{G} \rightarrow \mathrm{T}(\mathrm{E} 1-\mathrm{A} 66 \mathrm{~S}) ; 10201 \mathrm{G} \rightarrow \mathrm{A}$ (E1-D70N); $10660 \mathrm{~A} \rightarrow \mathrm{C}$ (E1-R223R)] from Clone\#1 virus were introduced into the pCHIKV-LR i.c. and resultant virus ( $\mathrm{LR}-\mathrm{Cl} \# 1$ ) was tested in standard and cholesterol-depleted C6/36 cells (Figure 5A, B). Growth kinetics of the i.c.-derived virus $\mathrm{LR}-\mathrm{Cl} \# 1$ in cholesteroldepleted C6/36 cells was indistinguishable from cholesterol-adapted virus (Clone\#1), and only slightly attenuated as compared to cholesterol-independent virus LR226A (Figure 5), thereby confirming the role of [E1A66S and E1-D70N] substitutions in modulating cholesterol dependence of CHIKV. One of these mutations [10660 A $\rightarrow$ C (E1-R223R)] does not lead to an amino acid substitution; however, it is located in close proximity to position E1-226. This mutation was present in all three individual clones (\#1, \#5 and \#7), suggesting that it might be important for modulating cholesterol dependence of CHIKV (Additional file 1, Table S4). To test this possibility, the point mutation $(10660 \mathrm{C} \rightarrow \mathrm{A})$ from the pLR-ApaI-226V was introduced into pLR-Cl\#1, and resultant virus (pLR-Cl\#1-10660A) was analyzed in standard and cholesterol-depleted C6/36 cells (Figure 5). This virus replicated as efficiently as $\mathrm{LR}-\mathrm{Cl} \# 1$ or Clone\# 1 viruses in cholesterol-depleted C6/36 cells
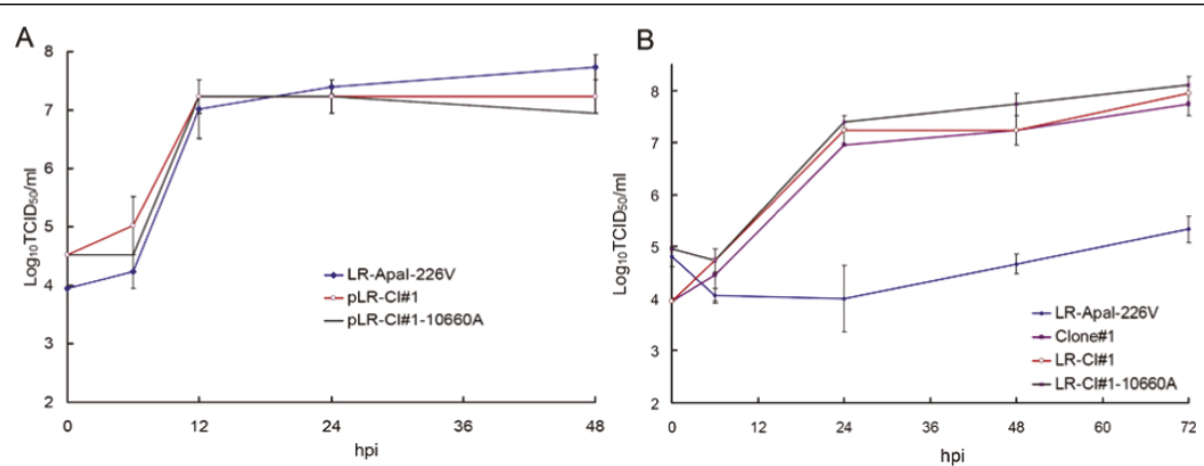

Figure 5 Growth kinetics of CHIKV with mutations in E1 protein identified in Clone\#1 virus in standard (A) and cholesterol-depleted (B) C6/36 cells. Standard cells were infected at an $\mathrm{MOI}$ of 1.0. Cholesterol-depleted cells were infected at an $\mathrm{MOI}$ of 0.1 . Viral titers are expressed as $\log _{10} T C I D_{50} / \mathrm{mL} \pm$ standard deviation of 2 independent experiments. 
indicating that the silent mutation $10660 \mathrm{~A} \rightarrow \mathrm{C}$ is not implicated in cholesterol sensitivity of CHIKV.

The eGFP-expressing version of LR-Cl\#1-10660A (LRGFP-Cl\#1-10660A) was constructed and specific infectivity of resultant virus was determined in standard and cholesterol-depleted C6/36 cells (Additional file 1, Figure S1). The LR-Cl\#1-10660A virus was able to infect cholesterol-depleted C6/36 cells significantly more efficiently ( $\mathrm{p}<0.01$ Student's $\mathrm{t}$ test) as compared to LRGFP-226V virus, which genetically corresponds to LRApaI-226V virus, used for the adaptation experiment.

To evaluate if the roles of the mutations [E1-A66S and E1-D70N] from Clone \#1 virus in modulating cholesterol dependence of CHIKV were correlated with the role of these mutations in CHIKV infectivity to $A e$. albopictus midguts, the $\mathrm{OID}_{50}$ value of LR-GFP-Cl\#110660A was determined as described above (Table 1). The infectivity of LR-GFP-Cl\#1-10660A virus was almost identical to that of LR-GFP-226V ( $p>0.1$ ), indicating that [E1-A66S and E1-D70N] mutations do not affect mosquito infectivity of CHIKV. These data also indicate that cholesterol dependence of CHIKV does not directly correlate with increased mosquito infectivity.

\section{E1-A226V mutation is responsible for decrease in $\mathrm{pH}$ threshold for fusion reaction}

The $\mathrm{pH}$ dependence of the fusion reaction can be analyzed by monitoring the role of agents that act to raise the $\mathrm{pH}$ of the endosome in inhibition of viral entry into the cells [34]. The lysotropic agent ammonium chloride $\left(\mathrm{NH}_{4} \mathrm{Cl}\right)$ has been used previously to demonstrate the effect of mutations in both SFV and SINV on their fusion thresholds $[35,36]$. The LR-ApaI-226V and LR$226 \mathrm{~A}$ viruses were mixed in a $1: 1$ ratio and used to infect $\mathrm{C} 6 / 36$ or BHK-21 cells pretreated with different concentrations of $\mathrm{NH}_{4} \mathrm{Cl}$. At 48 hpi cell culture supernatants were collected and either titrated on Vero cells or used for RNA extraction followed by RT-PCR and ApaI restrictase digestion to determine the relative RNA ratio of competing viruses. LR-ApaI-226V was markedly more sensitive to inhibition with $\mathrm{NH}_{4} \mathrm{Cl}$ than was LR$226 \mathrm{~A}$ virus. Thus in $\mathrm{C} 6 / 36$ cells in the presence of 3,6 , and $9 \mathrm{mM}$ of $\mathrm{NH}_{4} \mathrm{Cl}$, the majority of CHIKV RNA in the supernatant was derived from LR-226A virus (Figure 6A). Similarly, in BHK-21 cells, LR-226A virus outcompeted LR-ApaI-226V at $\mathrm{NH}_{4} \mathrm{Cl}$ concentrations of 9 and $12 \mathrm{mM}$ (Figure 6B). These data suggest that CHIKV with the E1-A226V mutation requires a low $\mathrm{pH}$ to trigger the fusion reaction. To confirm these findings, $\mathrm{pH}-$ dependence of the cell-cell fusion was compared for LRGFP-226A and LR-GFP-226V viruses. In two independent experiments the LR-GFP-226V virus triggered cellcell fusion reaction at $\sim 0.2$ lower $\mathrm{pH}$ as compared to LR-GFP-226A virus (Figure 7).

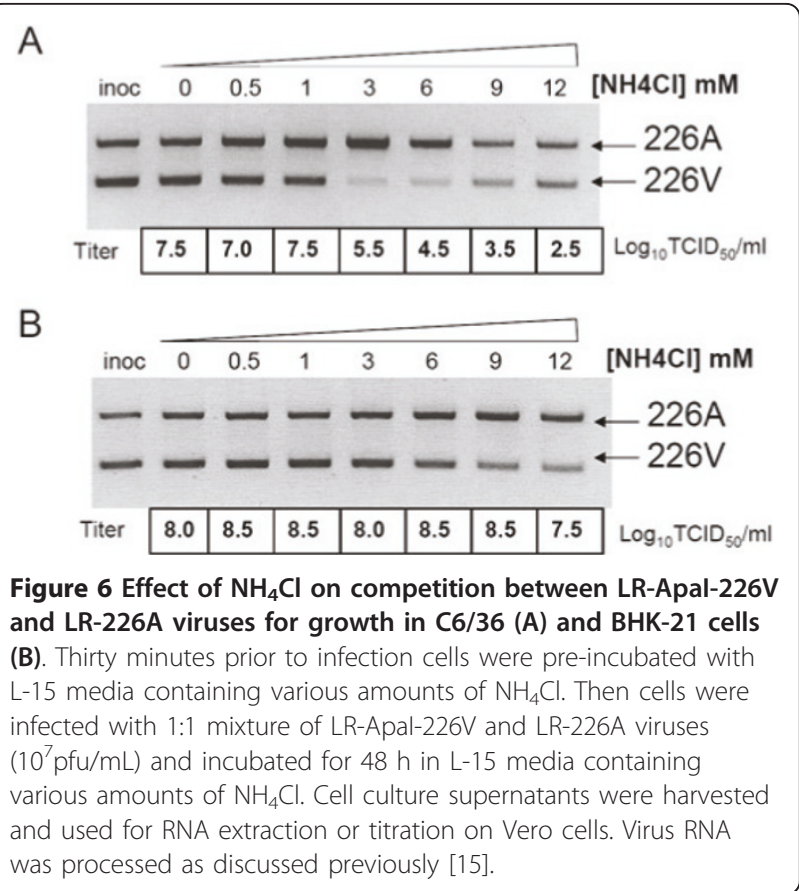

To investigate if CHIKV mosquito infectivity phenotypes correlated with different thresholds for their fusion reactions, cell-cell fusion assays were conducted for eGFP-expressing viruses with $\mathrm{S}, \mathrm{T}, \mathrm{G}, \mathrm{I}, \mathrm{P}, \mathrm{F}, \mathrm{M}$ and $\mathrm{H}$ residues at E1-226 (Figure 8A). No apparent correlation was found between these two parameters.

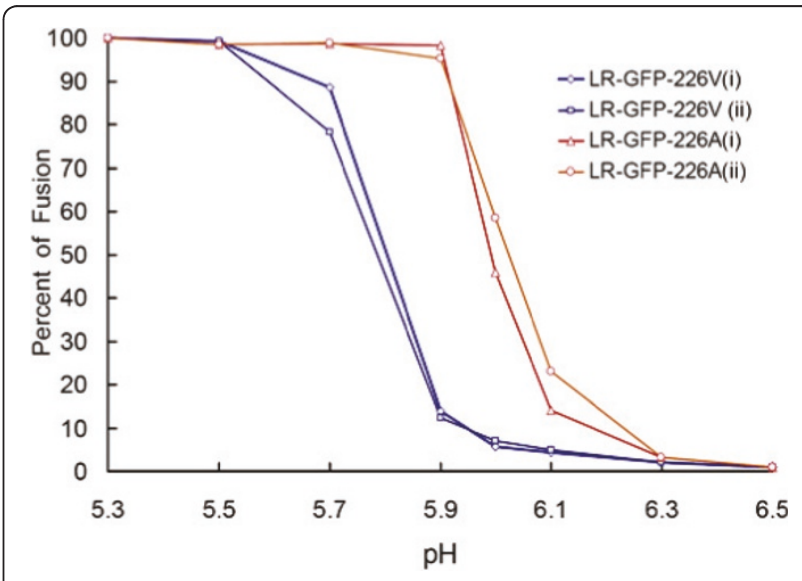

Figure 7 Effect of E1-A226V mutation on $\mathrm{pH}$ dependence of CHIKV induced cell-cell fusion. C6/36 cells infected with LR-GFP226A or LR-GFP-226V viruses were incubated for 2 min with L-15 medium whose $\mathrm{pH}$ was previously adjusted to desired values. The reaction was abrogated by replacement of the fusion medium with $0.5 \mathrm{~mL}$ of standard L-15 medium. Percent of fusion was calculated as $(1-\mathrm{c} / \mathrm{n}) \times 100 \%$, where $\mathrm{c}$ is a number of eGFP expressing cells, $\mathrm{n}$ is number of nuclei $(n \geq 70)$. Two independent experiments were performed for each virus ( $i$ and ii). Each individual experiment was performed in duplicates and results are expressed as an average of duplicates. 

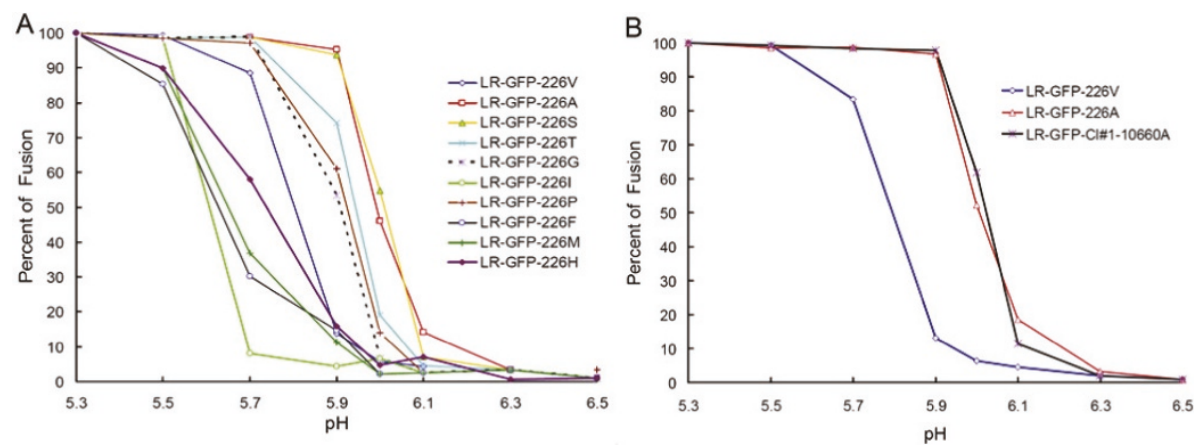

Figure 8 Effect of mutations at E1-226 (A) and [E1-66/E1-70] (B) on pH dependence of CHIKV induced cell-cell fusion. C6/36 cells were infected with eGFP expressing viruses and processed as described in legend for Figure 7. Experiments were performed in duplicates for each virus, and results are expressed as an average of duplicates.

Thus, mosquito infectivity of LR-GFP-226F virus was found to be similar to the infectivity of LR-GFP-226A virus (Table 1), however, LR-GFP-226F virus triggered the cell-cell fusion reaction at a $\mathrm{pH}$ even lower than the $\mathrm{pH}$ of fusion of the highly infectious virus LRGFP-226V ( $\mathrm{pH} \sim 5.6$ vs $\mathrm{pH} \sim 5.8$ respectively). Interestingly, mosquito infectivity of LR-GFP-Cl\#1-10660A virus was identical to that of LR-GFP-226V (Table 1), however, LR-GFP-Cl\#1-10660A triggered fusion reaction in $\mathrm{C6} / 36$ cells at $\mathrm{pH}$ markedly higher than LRGFP-226V (Figure 8B).

\section{Discussion}

The detailed mutagenesis analysis of position E1-226 demonstrated that CHIKV can tolerate a significant variability of amino acids at position E1-226. Depending on specific residues, CHIKV exhibited various phenotypes in cell culture conditions. These phenotypes may be broadly categorized as follows (Additional file 1, Table S1, S2): 1) - viruses that have S, T, G, P were almost indistinguishable from parental (wild-type) virus containing $\mathrm{V}$ or $\mathrm{A}$; 2) - introduction of I, F, M or $\mathrm{H}$ residues resulted in viruses that were slightly attenuated; 3 ) - introduction of $L$ residues resulted in significant attenuation of CHIKV. Analysis of viruses with specific mutations at E1-226 in Ae. albopictus mosquitoes revealed that effects of these mutations can also be classified into three groups as follows: 1) - amino acids I, M and $L$ resulted in viruses that were highly infectious to midgut cells of Ae. albopictus. The $\mathrm{OID}_{50}$ values of these viruses were not significantly different from CHIKV with E1-226V; 2). Viruses with amino acids S, T, G or F at E1-226 were associated with a low infectivity phenotype for Ae. albopictus. The $\mathrm{OID}_{50}$ values of these viruses were statistically indistinguishable from the ancestral form of CHIKV with E1-226A residue. 3) - an intermediate infectivity phenotype was attributed to $\mathrm{P}$ and $\mathrm{H}$ residues. OID $_{50}$ values of these viruses were statistically higher than that of highly infectious viruses (V, I, M and L), but in the same time lower than that of low infectivity viruses (Table 1 ).

To investigate the effect of mutations at E1-226 on the CHIKV requirement for cholesterol, growth kinetics and specific infectivity were compared for CHIKV with different mutations at E1-226 in cholesterol-depleted C6/ 36 cells. The pre-epidemic CHIKV (E1-226A) infected and replicated much more efficiently in cholesteroldepleted C6/36 cells as compared to the mutated CHIKV (V, I, M, or L at E1-226) with a high infectivity phenotype in Ae. albopictus (Figure 1, 2, 3, 4). This observation suggests that CHIKV dependence for cholesterol might be associated with infectivity to Ae. albopictus. Interestingly, a recent study showed that the E1 protein of alphaviruses can directly bind to cholesterol, which influences fusogenic properties of the viruses [37]. This suggests that direct E1 binding to cholesterol can be responsible for an increase in CHIKV infectivity for Ae. albopictus. However, analysis of viruses that showed intermediate and decreased infectivity to Ae. albopictus does not support the conclusion that CHIKV dependence for cholesterol is mechanistically correlated with infectivity to Ae. albopictus. These viruses demonstrated a broad spectrum of cholesterol dependence phenotypes that do not correlate with mosquito infectivity profile. The simplest explanation for the lack of correlation is that dependence for cholesterol and infectivity to Ae. albopictus are two independent phenotypic effects of the E1-226 mutations. Alternatively, dependence for cholesterol might be controlled at several steps of the infectious process. Cholesterol-independent viruses (such as LR-226A and LR-226S) do not rely on cholesterol at each of these steps. However, cholesterol-dependent viruses (such as LR-226V and LR-226G) may require cholesterol at different (not overlapping) steps, and the CHIKV-cholesterol interaction at steps which are connected to Ae. albopictus infectivity are 
unnecessary for viruses that showed decreased infectivity for Ae. albopictus.

All of the residues at E1-226 that are associated with high infectivity for Ae. albopictus also demonstrated high dependence to cholesterol. To address the question if cholesterol dependence is absolutely required for high mosquito infectivity, we identified mutations in the CHIKV genome regions other than E1-226, which can make CHIKV less cholesterol-dependent (Figure 5, Additional file 1, Table S4). Introduction of E1-A66S and E1-D70N mutations into the i.c. of CHIKV with valine at E1-226 significantly releases cholesterol dependence of CHIKV; however, these mutations had no effect on CHIKV infectivity to Ae. albopictus mosquitoes. These data indicate that cholesterol dependence can be modulated independently of the phenotypes of infectivity to Ae. albopictus mosquitoes. Altogether, these observations and previously discussed data on the roles of individual mutations at E1-226 indicate that cholesterol dependence of CHIKV cannot serve as an indicator of infectious properties of CHIKV in $A e$. albopictus.

Interestingly, all substitutions that control cholesterol dependence of CHIKV occurred in an E1 region that has never previously been implicated in modulating cholesterol dependence of alphaviruses. Previously it was shown that besides the position E1-226, the mutations in the "hinge" region of the E1 protein (positions: E1-44 and E1-178) could also control cholesterol dependence of SFV. In addition, E1-44 and E1-178 mutations control SFV dependency for sphingolipids, and it was proposed that these mutations in the hinge region control relative flexibility of domain I and domain II of E1, which are believed to be important for the biological function of the molecule [38]. The effector mechanism of mutations from Clone\#1 (E1-A66S and E1-D70N) and Clone\#10 [(Q222H) data not shown] on CHIKV cholesterol dependence are unknown. The mutations E1-A66S and E1-D70N are located in the bc loop of domain II which is involved in interactions with Domain I of the adjacent E1 protein and E2 protein $[17,25]$. Therefore, it is possible that these mutations generally affect the stability of the CHIKV particle. The Q222H is located in the middle of the ij loop of E1 and probably acts by controlling the flexibility and the topology of the tip of this loop, which interacts with the fusion loop and target membrane.

The common feature shared by residues at E1-226 that ensure a high infectivity phenotype of CHIKV in Ae. albopictus (V, I, L and $\mathrm{M}$ ) is that they belong to class of non-polar aliphatic amino acids. Interestingly, all residues that do not fall into this category (A, S, T, G, F, P, H) were associated with significantly lower infectivity to Ae. albopictus. It appears that a general requirement for an increase in infectivity for Ae. albopictus is associated with the presence of at least two or more aliphatic carbon atoms connected to the $\beta$-carbon of alanine. The current model of the alphavirus membrane fusion process postulates that upon exposure to low $\mathrm{pH}$ and E2-E1 heterodimer dissociation, the fusion loop and/or adjacent ij loop (with position E1-226) act as "sensor of lipid composition" of target membranes, and then regulate the stable insertion of the fusion loop into membrane of endosomes $[26,39,40]$. Therefore, the aliphatic groups of V, I, M and L residues at the position E1-226 of ij loop could be directly involved in modulation of this "lipid sensor" step via direct interaction with target membrane or by changing conformation of the fusion loop, which is located in close proximity to the ij loop. However, we also cannot exclude the possibility that mutations at the tip of the ij loop (E1-226) act independently of the fusion loop by changing overall conformation of E1 protein during this "lipid sensing" step.

Competition experiments between LR-ApaI-226V and LR-226A in the presence of inhibitor of endosomal acidification $\left(\mathrm{NH}_{4} \mathrm{Cl}\right)$, and cell-cell fusion experiments demonstrated that the E1-A226V mutation is also responsible for a decrease in the $\mathrm{pH}$ required for triggering of a fusion reaction (Figure 6,7 ). The observed difference in $\mathrm{pH}$ dependence between LR-GFP-226V and LR-GFP-226A viruses was relatively small $(\sim 0.2)$; however, it is possible that it can have significant impact on the location of where the fusion reaction occurs within endosomal compartments. Thus, lower $\mathrm{pH}$ suggests that fusion of E1-226V viruses would occur not in early endosomes ( $\mathrm{pH}$ 6.5-6.0), but rather in late endosomes $(\mathrm{pH}<6.0)$, which have quite different lipid composition [41-44]. Interestingly, residues E1-226I, and E1$226 \mathrm{M}$ that were found to significantly increase CHIKV infectivity to Ae. albopictus, also led to a decrease in the $\mathrm{pH}$ of fusion reaction. However, the further analyses of the effects of different mutations at E1-226 on pH of fusion reaction failed to support the association between $\mathrm{pH}$-dependence of the fusion reaction and CHIKV phenotypes in Ae. albopictus (Figure 8). This indicates that a decrease in the $\mathrm{pH}$ of fusion reaction due to E1A226V mutation is probably not directly responsible for the observed increase in CHIKV infectivity to Ae. albopictus mosquitoes.

There was also no clear correlation between $\mathrm{pH}$ and cholesterol dependence of CHIKV. Thus, although it seems that viruses that have lower cholesterol dependence for cell entry have a tendency to trigger fusion at higher $\mathrm{pH}$, there are two exceptions that do not fit this relationship. The E1-226F mutation was responsible for intermediate dependency for cholesterol, but required very low $\mathrm{pH}$ to trigger fusion. However, CHIKV with 
E1-226G was highly cholesterol-dependent, but triggered fusion at markedly higher $\mathrm{pH}$ than the other highly cholesterol-dependent virus. Interestingly, the E1-P226S mutation in SFV which is also responsible for modulation of cholesterol dependence, has no effect on SFV pH dependence for cell entry [39].

It remains perplexing why wild-type isolates of some alphaviruses such a SFV and SINV are highly dependent on the presence of cholesterol in membranes of host cell [21,23], whereas others such as CHIKV [15] and VEEV [27] can resist cholesterol depletion? It is particularly surprising considering the fact that biological transmission of alphaviruses occurs in mosquito vectors that do not have enzymatic pathway to synthesize cholesterol and obtain required sterols directly from the diet $[45,46]$. Our data shows that dependence for cholesterol is not directly associated with mosquito infectivity or $\mathrm{pH}$ dependence phenotypes. Moreover, it was shown that a cholesterol-independent mutation E1-P226S in SFV confers more efficient viral replication in intrathoracicaly injected Ae. albopictus compared to highly cholesterol-dependent wild type SFV [47], however, selection of this mutation by SFV strains in nature has never been documented. Since mutations that control cholesterol dependence also control many other unrelated functions of the alphaviruses, we hypothesize that cholesterol dependence/independence is not associated with any specific evolutionary force that determines alphavirus evolution in nature, and is acquired/lost by particular viruses as a result of viral adaptation to other conditions/functions associated with specific host specie used by viruses in given ecological settings.

\section{Conclusions}

In summary, we demonstrated that mutation E1-A226V is associated with multiple effects on CHIKV physiology, such as cholesterol and $\mathrm{pH}$ dependency and mosquito infectivity. The detailed analyses of CHIKV with different mutations at E1-226 and other genome regions revealed that there is no clear correlation between these parameters. This indicates that the E1-A226V mutation probably acts at different steps of CHIKV life cycle affecting multiple functions of the virus.

\section{Additional material}

Additional file 1: Figure S1, Table S1, Table S2, Table S3 and Table S4. The file contains Figure S1, Table S1, Table S2, Table S3, Table S4 and legends for these Figure and Tables.

\section{Acknowledgements}

We are grateful to Jing Haung and Aysha Chatueva for their assistance with mosquito and cells culture experiments. We also thank Drs. Scott Weaver,
Margaret Kielian, Robert Tesh and Stanley Watowich for providing editorial comments and suggestions during the manuscript preparation. KAT was supported by McLaughlin Endowment fund, CEM was supported by Centers for Disease Control Grant for Public Health Research Dissertation (R36) PAR07-231. Funding for this study was provided by NIH R21 Al073389

\section{Author details}

'Department of Pathology, University of Texas Medical Branch, Galveston, Texas, USA. ${ }^{2}$ Carolina Vaccine Institute, University of North Carolina, Chapel Hill, North Carolina, USA.

\section{Authors' contributions}

KAT designed the experiments; KAT, CEM performed experiments, KAT, CEM and $\mathrm{SH}$ analyzed the data and wrote the paper. All authors read and approved the final manuscript.

\section{Competing interests}

The authors declare that they have no competing interests.

Received: 15 June 2011 Accepted: 29 July 2011 Published: 29 July 2011

\section{References}

1. Tsetsarkin KA, Chen R, Leal G, Forrester N, Higgs S, Huang J, Weaver SC: Chikungunya virus emergence is constrained in Asia by lineage-specific adaptive landscapes. Proc Natl Acad Sci USA 2011, 108:7872-7877.

2. Schwartz O, Albert ML: Biology and pathogenesis of chikungunya virus. Nat Rev Microbiol 2010, 8:491-500.

3. Rezza $G$, Nicoletti $L$, Angelini $R$, Romi $R$, Finarelli AC, Panning $M$, Cordioli $P$, Fortuna C, Boros S, Magurano F, et al: Infection with chikungunya virus in Italy: an outbreak in a temperate region. Lancet 2007, 370:1840-1846.

4. Grandadam M, Caro V, Plumet S, Thiberge JM, Souares $Y$, Failloux AB, Tolou HJ, Budelot M, Cosserat D, Leparc-Goffart I, Despres P: Chikungunya virus, southeastern france. Emerg Infect Dis 2011, 17:910-913.

5. Delatte H, Paupy C, Dehecq JS, Thiria J, Failloux AB, Fontenille D: [Aedes albopictus, vector of chikungunya and dengue viruses in Reunion Island: biology and control]. Parasite 2008, 15:3-13.

6. Ng LC, Tan LK, Tan CH, Tan SS, Hapuarachchi HC, Pok KY, Lai YL, LamPhua SG, Bucht G, Lin RT, et al: Entomologic and virologic investigation of Chikungunya, Singapore. Emerg Infect Dis 2009, 15:1243-1249.

7. Bonilauri $P$, Bellini R, Calzolari M, Angelini R, Venturi L, Fallacara F, Cordioli $P$, Angelini P, Venturelli C, Merialdi G, Dottori M: Chikungunya virus in Aedes albopictus, Italy. Emerg Infect Dis 2008, 14:852-854.

8. Kumar NP, Joseph R, Kamaraj T, Jambulingam P: A226V mutation in virus during the 2007 chikungunya outbreak in Kerala, India. J Gen Virol 2008, 89:1945-1948.

9. Hapuarachchi HC, Bandara KB, Sumanadasa SD, Hapugoda MD, Lai YL Lee KS, Tan LK, Lin RT, Ng LF, Bucht G, et al: Re-emergence of Chikungunya Virus in Southeast Asia: Virologic Evidence from Sri Lanka and Singapore. J Gen Virol 2009, 91:1067-1076.

10. Pages F, Peyrefitte CN, Mve MT, Jarjaval F, Brisse S, Iteman I, Gravier P, Tolou H, Nkoghe D, Grandadam M: Aedes albopictus mosquito: the main vector of the 2007 Chikungunya outbreak in Gabon. PLOS One 2009, 4: e4691.

11. Schuffenecker I, Iteman I, Michault A, Murri S, Frangeul L, Vaney MC, Lavenir R, Pardigon N, Reynes JM, Pettinelli F, et al: Genome microevolution of chikungunya viruses causing the Indian Ocean outbreak. PLoS Med 2006, 3:e263.

12. de Lamballerie X, Leroy E, Charrel RN, Ttsetsarkin K, Higgs S, Gould EA: Chikungunya virus adapts to tiger mosquito via evolutionary convergence: a sign of things to come? Virol J 2008, 5:33.

13. Sam IC, Chan YF, Chan SY, Loong SK, Chin HK, Hooi PS, Ganeswrie R, Abubakar S: Chikungunya virus of Asian and Central/East African genotypes in Malaysia. J Clin Virol 2009, 46:180-183.

14. Vazeille M, Moutailler S, Coudrier D, Rousseaux C, Khun H, Huerre M, Thiria J, Dehecq JS, Fontenille D, Schuffenecker I, et al: Two Chikungunya isolates from the outbreak of La Reunion (Indian Ocean) exhibit different patterns of infection in the mosquito, Aedes albopictus. PLOS One 2007, 2:e1168.

15. Tsetsarkin KA, Vanlandingham DL, McGee CE, Higgs S: A single mutation in chikungunya virus affects vector specificity and epidemic potential. PLOS Pathog 2007, 3:e201. 
16. Tsetsarkin K: Adaptation of chikungunya virus to aedes albopictus mosquitoes: the role of mutations in the e1 and e2 glycoproteins. The University of Texas Medical Branch, The University of Texas Graduate School of Biomedical Sciences; 2009

17. Voss JE, Vaney MC, Duquerroy S, Vonrhein C, Girard-Blanc C, Crublet E, Thompson A, Bricogne G, Rey FA: Glycoprotein organization of Chikungunya virus particles revealed by X-ray crystallography. Nature 2010, 468:709-712.

18. Strauss JH, Strauss EG: The alphaviruses: gene expression, replication, and evolution. Microbiol Rev 1994, 58:491-562.

19. Kielian M: Class II virus membrane fusion proteins. Virology 2006 344:38-47.

20. Phalen $T$, Kielian M: Cholesterol is required for infection by Semliki Forest virus. J Cell Biol 1991, 112:615-623.

21. Marquardt MT, Phalen T, Kielian M: Cholesterol is required in the exit pathway of Semliki Forest virus. J Cell Biol 1993, 123:57-65.

22. Vashishtha M, Phalen T, Marquardt MT, Ryu JS, Ng AC, Kielian M: A single point mutation controls the cholesterol dependence of Semliki Forest virus entry and exit. J Cell Biol 1998, 140:91-99.

23. LU YE, Cassese T, Kielian M: The cholesterol requirement for sindbis virus entry and exit and characterization of a spike protein region involved in cholesterol dependence. J Virol 1999, 73:4272-4278.

24. Gibbons DL, Erk I, Reilly B, Navaza J, Kielian M, Rey FA, Lepault J: Visualization of the target-membrane-inserted fusion protein of Semliki Forest virus by combined electron microscopy and crystallography. Cell 2003, 114:573-583.

25. Roussel A, Lescar J, Vaney MC, Wengler G, Wengler G, Rey FA: Structure and interactions at the viral surface of the envelope protein E1 of Semliki Forest virus. Structure 2006, 14:75-86.

26. Kielian M, Chatterjee PK, Gibbons DL, LU YE: Specific roles for lipids in virus fusion and exit. Examples from the alphaviruses. Subcell Biochem 2000, 34:409-455.

27. Kolokoltsov AA, Fleming EH, Davey RA: Venezuelan equine encephalitis virus entry mechanism requires late endosome formation and resists cell membrane cholesterol depletion. Virology 2006, 347:333-342.

28. Tsetsarkin K, Higgs S, McGee CE, De Lamballerie X, Charrel RN, Vanlandingham DL: Infectious clones of Chikungunya virus (La Reunion isolate) for vector competence studies. Vector Borne Zoonotic Dis 2006, 6:325-337

29. Higgs S, Olson KE, Kamrud Kl, Powers AM, Beaty BJ: Viral expression systems and viral infections in insects. In The Molecular Biology of Disease Vectors: A Methods Manual. Edited by: Crampton JM, Beard CB, Louis C. UK: Chapman 1997:457-483.

30. Weinstein DB: A single-step adsorption method for removal of lipoproteins and preparation of cholesterol-free serum. Circulation 1979, 60:204.

31. Lemm JA, Durbin RK, Stollar V, Rice CM: Mutations which alter the level or structure of nsP4 can affect the efficiency of Sindbis virus replication in a host-dependent manner. J Virol 1990, 64:3001-3011.

32. McElroy KL, Tsetsarkin KA, Vanlandingham DL, Higgs S: Role of the yellow fever virus structural protein genes in viral dissemination from the Aedes aegypti mosquito midgut. J Gen Virol 2006, 87:2993-3001.

33. Vanlandingham DL, Tsetsarkin K, Hong C, Klingler K, McElroy KL, Lehane MJ, Higgs S: Development and characterization of a double subgenomic chikungunya virus infectious clone to express heterologous genes in Aedes aegypti mosquitoes. Insect Biochem Mol Biol 2005, 35:1162-1170.

34. Kielian MC, Marsh M, Helenius A: Kinetics of endosome acidification detected by mutant and wild-type Semliki Forest virus. Embo J 1986, 5:3103-3109.

35. Glomb-Reinmund S, Kielian M: fus-1, a pH shift mutant of Semliki Forest virus, acts by altering spike subunit interactions via a mutation in the E2 subunit. J Virol 1998, 72:4281-4287.

36. Glomb-Reinmund S, Kielian M: The role of low $\mathrm{pH}$ and disulfide shuffling in the entry and fusion of Semliki Forest virus and Sindbis virus. Virology 1998, 248:372-381.

37. Umashankar M, Sanchez-San Martin C, Liao M, Reilly B, Guo A, Taylor G, Kielian M: Differential cholesterol binding by class II fusion proteins determines membrane fusion properties. J Virol 2008, 82:9245-9253.

38. Chatterjee PK, Eng CH, Kielian M: Novel mutations that control the sphingolipid and cholesterol dependence of the Semliki Forest virus fusion protein. J Virol 2002, 76:12712-12722
39. Chatterjee PK, Vashishtha M, Kielian M: Biochemical consequences of a mutation that controls the cholesterol dependence of Semliki Forest virus fusion. J Virol 2000, 74:1623-1631.

40. Gibbons DL, Ahn A, Liao M, Hammar L, Cheng RH, Kielian M: Multistep regulation of membrane insertion of the fusion peptide of Semliki Forest virus. J Virol 2004, 78:3312-3318.

41. Marsh M, Helenius A: Virus entry: open sesame. Cell 2006, 124:729-740.

42. Falguieres T, Luyet PP, Gruenberg J: Molecular assemblies and membrane domains in multivesicular endosome dynamics. Exp Cell Res 2009 315:1567-1573.

43. Sobo K, Chevallier J, Parton RG, Gruenberg J, van der Goot FG: Diversity of raft-like domains in late endosomes. PLoS One 2007, 2:e391.

44. Gruenberg J, van der Goot FG: Mechanisms of pathogen entry through the endosomal compartments. Nat Rev Mol Cell Biol 2006, 7:495-504.

45. Clayton RB: The Utilization Of Sterols By Insects. J Lipid Res 1964, 15:3-19.

46. Canavoso LE, Jouni ZE, Karnas KJ, Pennington JE, Wells MA: Fat metabolism in insects. Annu Rev Nutr 2001, 21:23-46.

47. Ahn A, Schoepp RJ, Sternberg D, Kielian M: Growth and stability of a cholesterol-independent Semliki Forest virus mutant in mosquitoes. Virology 1999, 262:452-456.

\section{doi:10.1186/1743-422X-8-376}

Cite this article as: Tsetsarkin et al:: Chikungunya virus adaptation to Aedes albopictus mosquitoes does not correlate with acquisition of cholesterol dependence or decreased $\mathrm{pH}$ threshold for fusion reaction. Virology Journal 2011 8:376.

\section{Submit your next manuscript to BioMed Central and take full advantage of:}

- Convenient online submission

- Thorough peer review

- No space constraints or color figure charges

- Immediate publication on acceptance

- Inclusion in PubMed, CAS, Scopus and Google Scholar

- Research which is freely available for redistribution

Submit your manuscript at www.biomedcentral.com/submit
C Biomed Central 